\title{
DO LOCAL AO GLOBAL: A EDUCAÇÃO CONTEXTUALIZADA E O DIÁLOGO INTERCULTURAL NA PROPOSTA DE INTERNACIONALIZAÇÃO DA EXTENSÃO INTEREURISLAND NO PPGESA
}

Nicola Andrian ${ }^{42}$

\begin{abstract}
Resumo
O artigo apresenta a pesquisa a partir da qual foi possível a realização do evento científico I Colóquio Brasil Itália. Uma pesquisa vinculada ao Programa de Pós-Graduação stricto sensu - Mestrado Multidisciplinar em Educação, Cultura e Territórios Semiáridos (PPGESA), Departamento de Ciências Humanas (DCH), Campus III da UNEB em Juazeiro, com bolsa de estudo do Programa Nacional de Pós-Doutorado (PNPD), Coordenação de Aperfeiçoamento de Pessoal de Nível Superior (CAPES). Através da pesquisa pretende-se analisar o conceito e a contribuição da educação contextualizada como foco de processos e práticas de diálogo intercultural, internacionalização e responsabilidade social da universidade que visam a educação das jovens gerações, frente as transformações e aos novos paradigmas pedagógicos, rumo a uma cidadania planetária. Um dos resultados esperados, através de um estudo de caso, é o de poder confirmar a tese segundo a qual a educação contextualizada é de grande relevância no diálogo intercultural a ser desenvolvido através dos processos e práticas de internacionalização da extensão do Ciclo INTEREURISLAND, entre o PPGESA, DCH III, UNEB e o Departamento FISPPA, da Universidade de Padova, Itália.
\end{abstract}

Palavras-chave: Educação contextualizada. Diálogo intercultural. Internacionalização. Responsabilidade social da universidade. Aprendizagem Solidária Internacional.

DAL LOCALE AL GLOBALE. L'EDUCAZIONE CONTESTUALIZZATA E IL DIALOGO INTERCULTURALE NELLA PROPOSTA DI INTERNAZIONALIZZAZIONE DELLA RESPONSABILITÀ SOCIALE DELL'UNIVERSITÀ INTEREURISLAND AL PPGESA

\section{Sintesi}

L'articolo presenta la ricerca a partire dalla quale è stato possibile organizzare l'evento scientifico I Colóquio Brasil Itália. Una ricerca vincolata al programma post-lauream Stricto Sensu - Master multidisciplinare in Istruzione, Cultura e Territori semi-aridi PPGESA, del Dipartimento di Scienze umane - DCH, UNEB Campus III a Juazeiro-BA, con una borsa di studio del Programma Nazionale di Post Dottorato - PNPD, del

\footnotetext{
42 Bolsista do Programa Nacional Pós-doutorado (PNPD), Coordenação de Aperfeiçoamento de Pessoal de Nível Superior (CAPES), vinculado ao Programa de Pós-graduação stricto sensu - Mestrado Multidisciplinar em Educação, Cultura e Territórios Semiáridos (PPGESA), Departamento de Ciências Humanas (DCH), Campus III da UNEB em Juazeiro-BA. Coordenador do Grupo permanente de internacionalização acadêmica DCH III / UNEB.
} 
Coordinamento per il miglioramento del personale dell'istruzione superiore - CAPES. Attraverso la ricerca si intende analizzare il concetto e il contributo dell'educazione contestualizzata come focus dei processi e delle pratiche di dialogo interculturale, internazionalizzazione e responsabilità sociale dell'università che mirano all'educazione delle giovani generazioni, di fronte alle trasformazioni e ai nuovi paradigmi pedagogici, verso una cittadinanza planetaria. Uno dei risultati attesi, attraverso un caso di studio, è quello di poter confermare la tesi secondo cui l'educazione contestualizzata è di grande rilevanza nel dialogo interculturale da sviluppare attraverso i processi e le pratiche di internazionalizzazione della responsabilità sociale della ciclicità INTEREURISLAND, tra il PPGESA, DCH III, della UNEB e il Dipartimento FISPPA, dell'Università di Padova, Italia.

Parole chiave: Educazione Contestualizzata. Dialogo interculturale. Internazionalizzazione. Responsabilità sociale universitaria. Service Learning Internazionale.

\title{
FROM THE LOCAL TO THE GLOBAL: CONTEXTUALIZED EDUCATION AND INTERCULTURAL DIALOGUE IN THE PROPOSAL FOR INTERNATIONALIZATION OF UNIVERSITY SOCIAL RESPONSIBILITY INTEREURISLAND AT PPGESA
}

\begin{abstract}
The article presents the research from which it was possible to organize the scientific event I Colóquio Brasil Itália. Research linked to the post-graduate program Stricto Sensu - Multidisciplinary Master in Education, Culture and Semi-arid Territories - PPGESA, of the Department of Human Sciences - DCH, UNEB Campus III in Juazeiro-BA, with a scholarship from the Postdoctoral National Programme - PNPD, Coordination for the Improvement of Higher Education Staff - CAPES. Through research we intend to analyse the concept and contribution of contextualized education as a focus of the processes and practices of intercultural dialogue, internationalization, and social responsibility of the university. Processes and practices that aim to educate the younger generations, in face of transformations and new pedagogical paradigms, towards a planetary citizenship. One of the expected results, through a case study, is being able to confirm the thesis according to which contextualized education is of great importance in an intercultural dialogue to be developed through the processes and practices of internationalization of social responsibility of the INTEREURISLAND cyclicity, between the PPGESA, DCH III, of the UNEB and the FISPPA Department, of the University of Padua, Italy.
\end{abstract}

Keywords: Contextualized Education. Intercultural dialogue. Internationalization. University social responsibility. International Service Learning. 


\section{Introdução}

O fenómeno da globalização, a abertura dos mercados nacionais para horizontes internacionais, diferentes níveis de progresso social com uma aceleração das trocas de informações em função das novas tecnologias e fluxos migratórios muitas vezes forçados, para pesquisadores de diferentes áreas, entre os quais a Galeri (2009, p.98), foram as causas que determinaram, no século passado, uma desorientação a nível político, econômico, científico e cultural. Estas novas condições de vida, principalmente nas grandes cidades e, em seguida, nos contextos rurais da grande maioria dos países nos cinco continentes, determinou, por um lado, o impulso para a unificação dos processos socioeconómicos e a universalização dos saberes e, por outro lado, a procura de identidade pessoal e senso de pertencer ao próprio contexto específico, à própria comunidade.

Novos e urgentes desafios foram postos, por isso, às instituições educativas de qualquer ordem e nível e em qualquer contexto, a respeito da educação e da formação do cidadão contemporâneo. Direcionando o olhar no contexto específico do semiárido brasileiro, segundo Reis (2010, p.10), faz se necessário defender

[...] uma educação que traz nas suas práticas educativas o desafio de exercitar a contextualização e interdisciplinaridade como estratégia para contrapor-se aos males da pedagogia moderna que se pautou pelos princípios da neutralidade, da formalidade abstrata e da universalidade dos saberes e das práticas.

Uma educação que compreende que "todo saber é singularizado em cada sujeito a partir de suas referências e que, portanto, todo saber é local" (Idem). Os sujeitos constroem os seus conhecimentos a partir das experiências vivenciadas diretamente, das relações criadas através de trocas diárias de saberes práticos e teóricos e da rede que eles vão tecendo no próprio contexto. Mas até quando a educação escolar 'formal' é uma educação descontextualizada e, como ressalta Martins (2006),

por sê-lo, é também colonizadora, ou seja, ela se dirige hegemonicamente de uma determinada realidade - atualmente majoritariamente esta realidade é a do sudeste urbano do Brasil - e, a partir desta "sua realidade" e de uma narrativa pronunciada por um tal sujeito universal e abstrato denominado "nós brasileiros",

tal educação, de uma certa forma imposta por sujeitos que não conhecem eu/ou não interagem com os diferentes contextos, "toma todas as outras realidades que compõem a 
imensa diversidade brasileira, como sendo seus "Outros": "eles", "aqueles" que estão "lá" e devem ser integrados à sua narrativa.”. A 'Educação Contextualizada' específica do Semiárido Brasileiro, precisa

[...] abordar as particularidades do semi-árido - a questão climática, o problema das secas, a questão hídrica vista sob o prisma global e local, os aspectos culturais e simbólicos presentes - e a necessidade de a educação escolar se relacionar de forma mais efetivas com estes muitos problemas. Discutindo e rediscutindo o papel da educação contextualizada diante das transformações e dos novos paradigmas pedagógicos que apontam o destino do homen contemporâneo como um destino comum, um destino planetário. (KÜSTER, 2014, p.21)

Reconhecendo a importância de uma educação que se fundamenta no próprio contexto, como ponto de partida e de chegada dos conhecimentos e saberes diversos, a pedagogia precisa encarar o desafio de abrir o horizonte para 'o outro lado da moeda', as novas dimensões globais. Portanto, como preconiza Reis (2010), a Educação Contextualizada e para a Convivência com o Semiárido Brasileiro "[...] não pode ser entendida como o espaço do aprisionamento do conhecimento e do saber, ou ainda na perspectiva de uma educação localista, mas como aquela que se constrói no cruzamento cultura - escola - sociedade - mundo".

Tanto o global quanto o local são parte de um novo cenário educacional e a educação, na perspectiva da sustentabilidade, deve atuar em dupla escala, apoiando tanto a redescoberta e a valorização da dimensão local, quanto a dimensão global da sociedade. Para sublinhar a complementaridade entre fenômenos locais e globais, o sociólogo britânico Robertson Roland, em 1992, apresentou ao mundo o termo Glocalização ${ }^{43}$. Para a pesquisadora Galeri (2009, p.98)

Em projetos educacionais sustentáveis, devemos tentar superar a dicotomia local / global, pois a exploração da sala torna-se, para os estudantes, um primeiro campo de pesquisa para ativar e experimentar caminhos de pesquisa-ação, adquirir habilidades e desenvolver a sensação de cuidar do território de pertencimento. O local é o primeiro passo para alcançar uma consciência planetária como um objetivo para impulsionar a sustentabilidade educacional.

\footnotetext{
${ }^{43}$ ROLAND, R. Globalization: Social Theory and Global Culture. London: SAGE Publications, 1992.
} 
Considerando que pela UNESCO (2006), Cidadania global significa “[...] um sentimento de pertença a uma comunidade mais ampla e uma humanidade compartilhada, interdependência política, econômica, social e cultural e uma mistura de local, nacional e global ", e mantendo a abertura do horizonte entre o Local e o Global, é oportuno refletir sobre as considerações apresentadas no Livro Branco sobre o Diálogo Intercultural (EUROPEAN COUNCIL, 2008, pp. 10, 11) segundo as quais:

As abordagens tradicionais para gestão da diversidade cultural não são mais adequadas às sociedades que apresentam um nível sem precedentes de diversidade e em constante desenvolvimento - e quando - portanto, precisamos implementar uma nova estratégia para chegar à uma sociedade inclusiva: o diálogo intercultural.

Reconhecendo o diálogo e a relação autêntica como elementos fundamentais de cada 'encontro' com o outro, que em todo caso é diferente de mim, faz-se necessário, então, explorar novas possibilidade para que não nos isolemos do contato com o outro para salvaguardar nossa identidade cultural e não nos comprometermos com a diferença que nos incomoda. Para Freire P. (2005), respeito e curiosidade são elementos que nos possibilitam exercitar, aos poucos e diante do diferente, as vias de comunicação que podem nos unir sem que cada um perca suas características fundamentais. Uma postura que exige atenção na atitude de diálogo com o diferente que precisa ser visto em seus próprios termos. Tal vez os termos da Glocalização que poderá nos levar do contexto para o mundo e vice-versa.

[...] o índio não optou por pescar flechando. O seu estágio cultural e econômico, social etc. é esse, o que não significa que ele não saiba, que não possa saber de coisas que se dera fora desse estágio cultural. Então eu acho que o meu respeito da identidade cultural do outro exige de mim que eu não pretenda impor ao outro uma forma de ser de minha cultura, que tem outros cursos, mas também o meu respeito não me impõe negar ao outro o que a curiosidade do outro e o que ele quer saber mais daquilo que sua cultura propõe.(FREIRE, 2005, p.83)

Por todas as razões acima apresentadas, o contexto educacional escolhido para a presente pesquisa foi o da educação contextualizada em relação à implementação de práticas e processos inovadores de internacionalização da extensão universitária. Para tanto, delimitou-se como campo de estudo a implementação das novas estratégias do 
projeto INTEREURISLAND, no PPGESA, no Departamento de Ciências Humanas, Campus III, UNEB em Juazeiro-BA, no período entre maio de 2018 e abril de $2019 .{ }^{44}$

Atribuiu-se a escolha do contexto educacional e do período acima citados aos resultados obtidos através de uma pesquisa de doutorado em cotutela entre a Universidade de Padova, Itália, e a UNEB cujo título foi: INTEREURISLAND - De uma pesquisa no campo a uma possível referência para a internacionalização de experiências de responsabilidade social da Universidade.

Através de um estudo de caso piloto, a pesquisa analisou um ciclo completo do 'Progetto BEA', um projeto social e de intercâmbio que promove, para estudantes do curso de graduação em Ciências da Educação e da Formação da Universidade de Padova, experiências de mobilidade internacional com percursos mistos de estudo e estágio no exterior $^{45}$. Um sucessivo estudo de casos múltiplos analisou a implementação de novas estratégias de intercâmbio, derivadas do estudo de caso piloto, nos contextos de Rovigo, na Itália, e Juazeiro, no estado da Bahia, no Brasil, como polos de uma colaboração entre o Departamento de Filosofia, Sociologia, Pedagogia e Psicologia Aplicada da Universidade de Padova e o Departamento de Ciências Humanas, Campus III da UNEB. O Ciclo INTEREURISLAND propõe uma sequência de práticas nas três áreas principais: internacionalização e diálogo intercultural, intersetorialidade e responsabilidade social universitária e aprendizagem através do serviço solidário. Três áreas que se desenvolvem em reciprocidade e absoluta permeabilidade, cada uma partindo de e através das outras, seguindo uma sequência concêntrica. Considerando o que foi realizado através do Doutorado em cotutela e o que pretende-se desenvolver através da bolsa de PósDoutorado pelo PPGESA, vale ressaltar a importância da implementação de práticas de internacionalização, tanto no contexto de Padova e Rovigo, na Itália, pela União Europeia, quanto no contexto de Juazeiro-BA, pela CAPES (2017, p.6)

[...] a Capes, por meio da Diretoria de Relações Internacionais, pretende lançar as bases para novo programa fundamentado na realidade das instituições de ensino superior brasileiras (IES), que permita seu empoderamento por meio da promoção e fortalecimento de suas estratégias de internacionalização. É importante tornar as IES brasileiras mais proativas no seu processo de internacionalização, para que possam beneficiar-se ainda mais da expansão do seu impacto institucional e da apropriação dos conhecimentos adquiridos no exterior.

\footnotetext{
44 Tendo em conta a possibilidade de renovar por mais 12 meses o projeto de pesquisa apresentado.

${ }^{45}$ Mais informações a respeito do Progetto BEA são disponíveis no site

http://www.enars.it/joomla/it/com-joomgallery-image-manager/progetti/progetto-bea
} 
No parágrafo da Metodologia, serão apresentadas as atividades específicas de cada área do Ciclo INTEREURISLAND, para ampliar o entendimento da metodologia a ser implementada, tendo em vista seu desenvolvimento e contribuição para a pósgraduação do DCH III.

A Figura 1 apresenta o ciclo completo de atividades que os estudantes envolvidos seguem para o desenvolvimento de experiências de mobilidade internacional, com um sistema misto de estudo e estágio. O plano considera as atividades do semestre anterior ao período de mobilidade, realizado na universidade de origem, as atividades do semestre de mobilidade na universidade de acolhimento e as do semestre seguinte à mobilidade, de retorno à universidade de origem. Acha-se interessante ressaltar que para todas as atividades acima mencionadas encontra-se correspondência no documento da CAPES (Idem, p.7) a respeito do novo programa de internacionalização.

Para esse novo programa, a proposta é que as IES definam seus parceiros nacionais e internacionais e apresentem suas propostas de internacionalização. Essas deverão contar com infraestrutura para internacionalização, utilização de idiomas estrangeiros, escritório de relações internacionais, projetos para receber estudantes/pesquisadores estrangeiros, treinamento da sua equipe técnica, apropriação do conhecimento adquirido pelo bolsista após o retorno ao país, entre outras ações, que vão além da mobilidade ativa e passiva de docentes e discentes, construindo um ambiente internacional no dia a dia da Universidade.

Figura n.2: INTEREURISLAND - plano de mobilidade estudantil

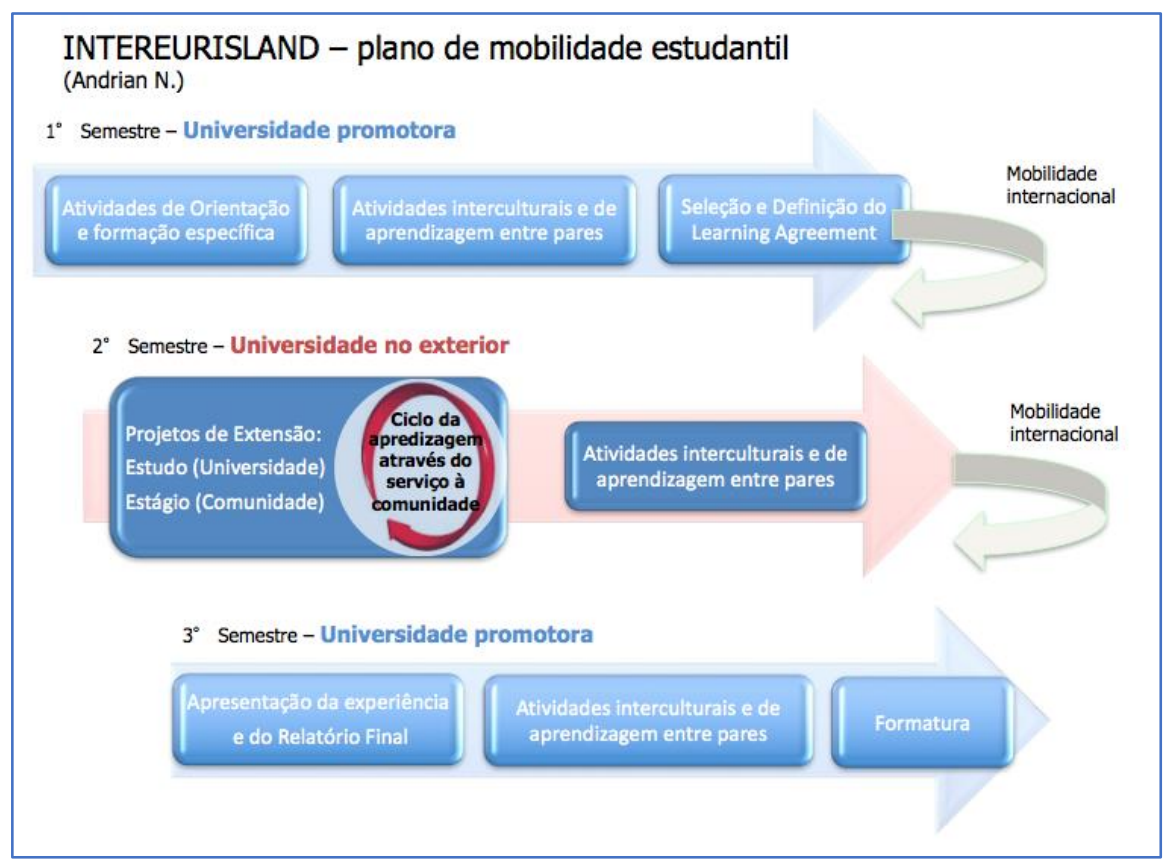

Fonte: Andrian N. (2017, pag.57) 
$\mathrm{Na}$ figura n.3 é apresentado o núcleo central, o 'Coração' da proposta INTEREURISLAND, que propõe a intersecção entre as práticas de internacionalização, de extensão e de aprendizagem através da chamada aprendizagem solidária. Vale neste momento ressaltar a importância também do desenvolvimento de práticas de extensão, tanto em Padova, Itália, como em Juazeiro-BA.

Figura n. 3: INTEREURISLAND, esquema de referência para projetos de extensão

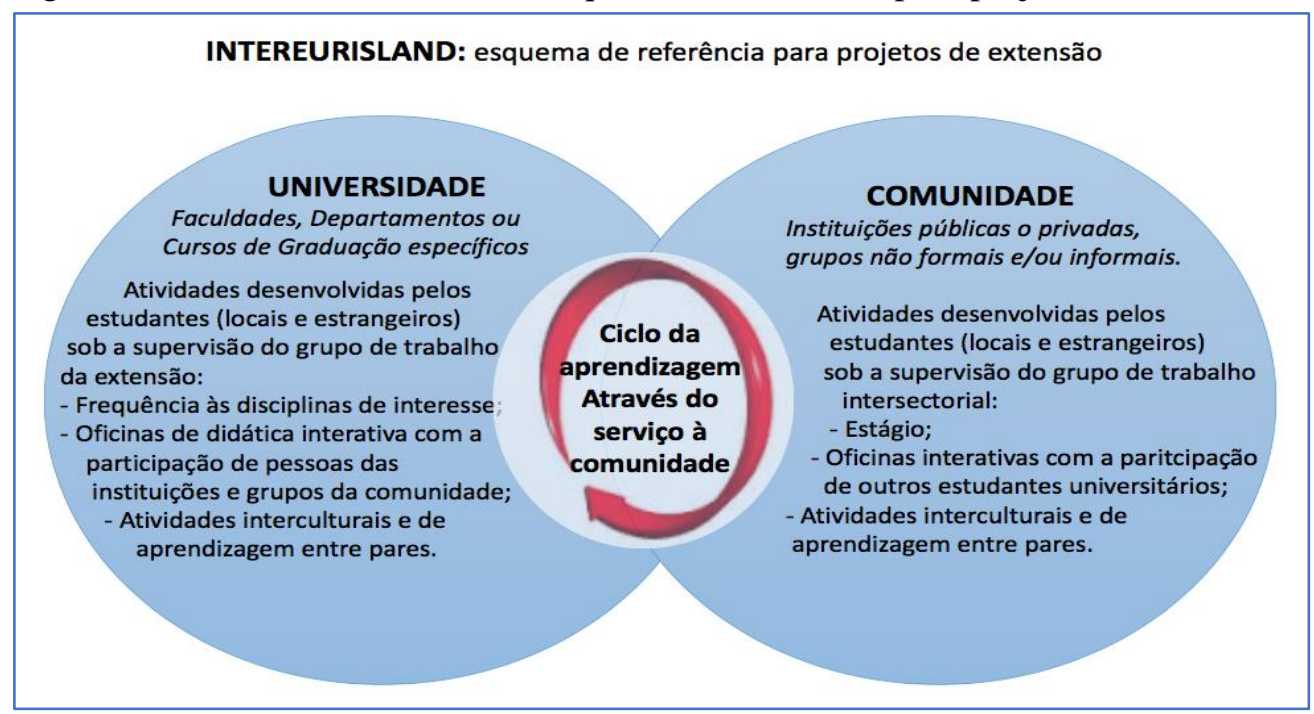

Fonte: Andrian N. (2017, pag.60)

\section{QUESTÕES DE PESQUISA}

O presente projeto apresenta duas questões de pesquisa como a seguir:

1) Quantas e quais práticas do Ciclo INTEREURISLAND (diálogo intercultural, internacionalização, extensão e aprendizagem através do serviço à comunidade com foco na educação contextualizada) serão implementas no curso de Mestrado Multidisciplinar em Educação, Cultura e Territórios Semiáridos PPGESA, ofertado pelo DCH III, UNEB, Juazeiro-BA, em colaboração com o Departamento de Filosofia, Sociologia, Pedagogia e Psicologia Aplicada da Universidade de Padova, Itália, no período entre Junho de 2018 e Maio de 2019?

2) Qual é a relevância, se tem, da educação contextualizada no desenvolvimento do diálogo intercultural e de uma educação GloCal (do local para o global) e viceversa, através da implementação das práticas do ciclo INTEREURISLAND (diálogo intercultural, internacionalização, extensão e aprendizagem através do 
serviço solidário com foco na educação contextualizada) no curso de Mestrado Multidisciplinar em Educação, Cultura e Territórios Semiáridos PPGESA, ofertado pelo DCH, Campus III, UNEB, Juazeiro-BA, em colaboração com o Departamento de Filosofia, Sociologia, Pedagogia e Psicologia Aplicada da Universidade de Padova, Itália?

\section{OBJETIVOS}

\subsection{OBJETIVO GERAL}

Implementar e analisar os processos e as práticas do ciclo INTEREURISLAND (diálogo intercultural, internacionalização, extensão e aprendizagem através do serviço solidário com foco na educação contextualizada) no curso de Mestrado Multidisciplinar em Educação, Cultura e Territórios Semiáridos PPGESA, ofertado pelo DCH, Campus III, UNEB, Juazeiro-BA, em colaboração com o Departamento de Filosofia, Sociologia, Pedagogia e Psicologia Aplicada da Universidade de Padova, Itália.

\subsection{OBJETIVOS ESPECÍFICOS}

3.2.1 De Implementação - Ação

- Articular a implementação das práticas e dos processos do Ciclo INTEREURISLAND, com foco na educação contextualizada, no curso de Mestrado Multidisciplinar em Educação, Cultura e Territórios Semiáridos PPGESA ofertado pelo DCH, Campus III, UNEB, Juazeiro-BA em colaboração com o Departamento de Filosofia, Sociologia, Pedagogia e Psicologia Aplicada da Universidade de Padova, Itália;

- Desenvolver os vínculos entre o DCH Campus III, UNEB, Juazeiro-BA e o Departamento FISPPA da Universidade de Padova, Itália para atividades de intercâmbio tanto a nível de graduação como de Pós-Graduação;

- Contribuir com as atividades acadêmicas do DCH, Campus III e no específico do curso de Pós-Graduação em convivência com o semiárido;

- Contribuir na implementação de práticas e processos de internacionalização do novo Núcleo de internacionalização do DCH campus III, UNEB, Juazeiro-BA, tais quais:

$\checkmark$ Desenvolvimento dos vínculos entro o DCH Campus III, UNEB, Juazeiro-BA e o Departamento FISPPA da Universidade de Padova, Itália para atividades de intercâmbio tanto a nível de graduação como de Pós-Graduação, com a 
possibilidade de abrir a rede internacional ao Curso de estudos e formação Juvenil da Universidade TANGAZA COLLEGE, de Nairobi, no Kenya, Africa;

$\checkmark$ Desenvolvimento da colaboração com a Associação de Promoção Social En.A.R.S. de Padova e com as atividades do Progetto $B E A^{46}$ realizadas na cidade de Petrolina-PE;

$\checkmark$ Articulação de uma proposta de implementação das práticas e dos processos do ciclo INTEREURISLAND em uma rede mundial vinculada ao programa CATTEDRE SCHOLAS da Fundação Pontifícia SCHOLAS OCCURRENTES, impulsionada pelo Papa Francisco ${ }^{47}$;

- Contribuir com novas propostas de intercambio e internacionalização universitária através de novas estratégias de abordagem do conceito GloCal (do contexto para o mundo).

\subsubsection{De pesquisa}

- Aprofundar o conhecimento teórico e prático sobre a educação contextualizada para a convivência com o semiárido e sobre a educação GloCal - do local para o global;

- Aprofundar o conhecimento teórico sobre a proposta pedagógica de Papa Francisco e prático a respeito do desenvolvimento da rede mundial do programa CATTEDRE SCHOLAS, como "espaço acadêmico" da Fundação Pontifícia SCHOLAS OCCURRENTES;

- Analisar as possíveis características comuns entre a educação contextualizada e a educação GloCal através de uma abordagem intercultural;

- Analisar a implementação das práticas e dos processos do ciclo INTEREURISLAND com foco na educação contextualizada, no curso de Mestrado Multidisciplinar em Educação, Cultura e Territórios Semiáridos PPGESA ofertado pelo DCH, Campus III, UNEB, Juazeiro-BA em colaboração com o Departamento de Filosofia, Sociologia, Pedagogia e Psicologia Aplicada da Universidade de Padova, Itália;

- Investigar a relevância da educação contextualizada no desenvolvimento da educação GloCal e vice-versa, tanto no PPGESA, como no DCH III, com discentes e docentes

\footnotetext{
${ }^{46}$ Projeto social e de intercâmbio, promovido pela En.A.R.S. Padova em colaboração com a Universidade de Padova, Itália e com o 'BEA, Centro de estudos e práticas' de Petrolina-PE (CNPJ 19.641.426/0001-52).

${ }^{47} \mathrm{http}: / /$ web.scholasoccurrentes.org.
} 
envolvidos nas atividades de intercâmbio e extensão (tanto brasileiros quanto italianos).

\title{
4 FUNDAMENTAÇÃO TEÓRICA
}

\subsection{EDUCAÇÃO CONTEXTUALIZADA PARA A COMVIVÊNCIA COM O SEMIÁRIDO}

Em uma primeira abordagem à temática da Educação Contextualizada para a convivência com o semiárido, percebe-se que para muitos autores (REIS, 2010; MARTINS, 2006; SENA, 2014; CRUZ, GURGEL, 2014), trata-se de um campo de pesquisa ainda em fase de pleno desenvolvimento. Entende-se que ainda há muito que se estudar e desvelar sobre o próprio contexto de interesse para, primeiramente, descontruir a ideia de que este é um lugar hostil, improdutivo. Uma ideia que está presente até em livros didáticos que carregam as opções político-filosóficas de quem os escreveu, como ressalta, Cruz e Gurgel (2014, p.34).

\begin{abstract}
Especificamente, no que tange à questão de constituição do espaço brasileiro, antes de olhar para a diversidade das regiões, boa parte dos livros didáticos contribui para a construção de paradigmas regionais que colocam a região Nordeste em desvantagem à região Sudeste, onde se localizam os grandes centros urbanos como São Paulo e Rio de Janeiro; favorecem historicamente a criação de estereótipos e discursos que apresentam impossibilidades de convivência com a região semiárida, visão que se estende às configurações sobre o Nordeste brasileiro.

A divulgação do flagelo da seca e da miséria fortaleceu esse discurso, causando impacto no plano nacional, bem como foi, por longos anos, e ainda o é, plataforma de muitos políticos e programas assistencialistas.
\end{abstract}

De fato, mesmo sendo difícil encontrar uma definição comum de Educação Contextualizada, reconhecida por todas/todos, os pesquisadores envolvidos neste processo de desvelamento, ao aprofundar a leitura e o estudo dos diferentes materiais produzidos (livros, artigos, cadernos multidisciplinares, projetos etc) entendem que existem múltiplos olhares a respeito desta abordagem educativa específica e que "a conversa sobre a educação no semiárido revela detalhes para além do nosso grande discurso que, muitas vezes, oculta gente e coisas que acontecem nesse espaço (PEREIRA, 2016, pag.11). Por Martins (2006), essa educação não chega a ser um conceito, mais sim

uma ideia, um discurso, uma perspectiva de qualificação de ações educativas que, em que pese a falta de uma definição mais 
precisa, não é uma coisa totalmente nova e já vem orientando práticas variadas no campo da educação, se desdobram no Semiárido Brasileiro (SAB), especialmente no setor nãogovernamental.

\subsection{GLOCALIZAÇÃO E EDUCAÇÃO GLOCAL}

"Glocalização" ou "glocalismo" é um termo formulado na década de 1980, em japonês (dochakuka). Em seguida foi traduzido para o inglês pelo sociólogo Roland Robertson e posteriormente elaborado pelo sociólogo Zygmunt Bauman para adaptar o panorama da globalização às realidades locais, com o interesse de estudar de uma forma melhor suas relações com os contextos internacionais.

Segundo Bauman (2005), "Globalização" e "localização" podem ser os dois lados da mesma moeda, tendo em conta que as duas partes da população mundial podem viver em lados diferentes. A glocalização inicia sua análise a partir de sistemas simples (locais) para chegar aos mais complexos (globais), enquanto a globalização parece favorecer sistemas complexos, muitas vezes ignorando as implicações nos contextos específicos.

Segundo a glocalização acredita-se que a fundação da sociedade. em todas as épocas, tem sido a comunidade local, a partir da interação dos indivíduos, organizada em grupos cada vez mais ampliados, presentes em um território. A organização desses grupos certamente constitui um conjunto de 'sistemas' que se tornam 'subsistemas' se estiverem relacionados a organizações mais complexas.

A glocalização coloca no centro de sua 'filosofia' o indivíduo, a pessoa humana, o patrimônio material e imaterial local da pessoa e do grupo ao qual pertencem. Não ignora a dialética que deriva do encontro-choque dos vários grupos dentro da lógica do sistema-subsistema, mas nunca perde de vista o micro (local) em sua relação com a macro (global).

Pensando ao âmbito acadêmico, nos últimos anos, houve um interesse crescente por um novo tipo de estudante internacional: o estudante 'glocal'. Os estudantes da Glocal foram definidos pelo Dr. Rahul Choudaha, diretor de Serviços de Pesquisa e Consultoria da World Education Services, como estudantes que têm aspirações globais, mas preferem permanecer em seus países ou regiões de origem para uma educação focada no próprio contexto, seguindo o lema das Nações Unidas: "Pense globalmente, aja localmente".

Um lema que tem um vínculo importante com o conceito de Cidadania global que, pela UNESCO (2018) "significa um sentimento de pertença a uma comunidade mais 
ampla e uma humanidade compartilhada, interdependência política, econômica, social e cultural e uma mistura de local, nacional e global.".

Ao refletir sobre essa relação entre o local e o global, apontando para uma abordagem focada no diálogo intercultural principalmente entre seres humanos, faz-se necessário refletir e conscientizar-se a respeito das polarizações, tão existentes quanto perigosas e desumanizadoras, tais quais, por exemplo: o norte do mundo desenvolvido e o sul subdesenvolvido; as potências (ou regiões) industriais e as sociedades agrícolas; os países imperialistas ou neocolonialistas e os países dominados economicamente e politicamente.

Ao considerar os contextos envolvidos na presente pesquisa é, também, de grande importância considerar e refletir constantemente sobre as hierarquizações a respeito de raça e gênero impostas pelo colonialismo e as repercussões que estas hierarquizações determinaram nas populações interessadas. Um exemplo concreto refere-se ao estado da Bahia, que, pela pesquisa Nacional por Amostra de Domicílios Contínua Anual, divulgada pelo Instituto Brasileiro de Geografia e Estatística/IBGE, no ano de 2019, apresenta o segundo maior número de população (por mil pessoas) e a maior variável de porcentagem da população de cor (ou raça) negra do Brasil (igual a $22.5 \%$ da população do estado $)^{48}$. A respeito desses dados, entre muitas possíveis reflexões sobre as repercussões das hierarquizações de raça e gênero, cita-se a de Ana Célia da Silva (2011), da Universidade Federal da Bahia, que ao apresentar a própria pesquisa, cujo objeto de investigação foi “[...] a representação social do negro no livro didático de Língua Portuguesa de Ensino Fundamental de $1^{\circ}$ e $2^{\circ} \operatorname{ciclos}^{\text {"49, }}$, relata que em pesquisas anteriores tinha identificado que “[...] os livros de Língua Portuguesa das mesmas séries e ciclos da década de 80 caracterizavam-se pela rara presença do negro, e essa rara presença era marcada pela desumanização e estigma" (Idem, p.13).

\subsection{INTERCULTURA}

Segundo a UNESCO (2006, p.15) a interculturalidade é um conceito dinâmico e refere-se à evolução das relações entre grupos culturais. Foi definida como "a existência e a interação equitativa de diferentes culturas e a possibilidade de gerar expressões culturais compartilhadas através do diálogo e do respeito.”. A interculturalidade mútua

\footnotetext{
${ }^{48}$ Dados disponíveis no link: https://sidra.ibge.gov.br/tabela/6408

${ }^{49}$ Livros didáticos da década de 90.
} 
pressupõe o multiculturalismo e se traduz em um intercâmbio e diálogo intercultural em nível local, regional, nacional ou internacional.

Interessante ressaltar a visão de Perotti (1994 in SANTERINI, 2017, p.88) segundo a qual, a interculturalidade (com sua abreviatura intercultura) pode, portanto, ser definida como o conjunto de processos (psíquicos, relacionais, institucionais) relativos às trocas e à relação dinâmica, não tanto entre culturas, entendidas como totalidades complexas, mas entre pessoas. A dimensão intercultural qualifica relações, educação ou formas políticas que enfatizam as relações (o prefixo inter) para superar uma lógica 'não' excluindo, em vez disso, a reciprocidade e a interdependência.

Em contextos cada vez mais multiculturais, a Pedagogia é chamada a se questionar sobre quais são as melhores formas de educação do cidadão contemporâneo. Uma educação que proteja a originalidade de todo ser humano, mas que esteja atenta à alteridade, à diversidade.

A cultura e a intercultura são temas centrais também da proposta de Paulo Freire (FREIRE, 1963, p.11), que afirma: "um outro dado que partíamos era o de que a educação trava uma relação dialética com a cultura. Desta forma a nossa ciência educativa não poderia sobrepor-se à realidade contextual nossa”. Uma cultura própria de cada sujeito, que, como resultado do seu trabalho, "do seu esforço criador e recriador", é reconhecida como ponto de partida de um diálogo significativo com o próprio contexto social,

Como imaginário criativo expressos na sua linguagem, muitas vezes sinônimos de resistência, bem como de subserviência, também culturalmente elaborados como forma de resignação histórica para assegurar sua sobrevivência diante da dominação cultural. (PEROZA, 2010, pag.4)

A partir desta conceptualização de cultura, a experiência da diversidade cultural em vários contextos no mundo leva o Paulo Freire a uma reflexão a respeito da intercultura e do impacto da sua obra no mundo e vice-versa: "estava, já na época, absolutamente convencido de quão útil e fundamental seria a mim correr mundo, exporme a contextos diversos, aprender das experiências de outros, rever-me nas diferenças culturais" (FREIRE \& FAUNDEZ, 1985, p. 12).

\section{METODOLOGIA}


Uma série de reflexões a respeito da implementação do Ciclo INTEREURISLAND no contexto da graduação no DCH III, da questão da pesquisa e dos objetivos apresentados, levaram-me a considerar o estudo de caso como a metodologia mais apropriada para este projeto de pesquisa porque é "uma investigação empírica que visa investigar um fenômeno contemporâneo dentro do seu contexto da vida real, especialmente quando os limites entre o fenômeno e o contexto não são claramente definidos" (YIN, 1994, p.32). O estudo de caso conduz os caminhos metodológicos, a serem seguidos, para a compreensão dos fenômenos individuas, organizacionais, sociais e políticos em um determinado contexto delimitado pelo tempo e pelo espaço. Por se tratar de um estudo local, sempre conforme Yin (Idem, p.21),

O estudo de caso permite uma investigação para se preservar as características holísticas e significativas dos eventos da vida real - tais como ciclos de vida individuais, processos organizacionais $\mathrm{e}$ administrativos, mudanças ocorridas em regiões urbanas, relações internacionais e a maturação de alguns setores.

Segundo vários autores (Smith N., 1990, Stake 1995, Yin, 2005), existem pelo menos cinco aplicações diferentes para um estudo de caso:

1. explicar os elos causais nas manifestações da vida real, demasiado complexas para a investigação ou para estratégias experimentais (estudo explicativo);

2. descrever uma intervenção e o contexto da vida real em que ela aconteceu (estudo de caso descritivo);

3. ilustrar determinados temas, sujeitos a avaliação, tanto de uma perspectiva descritiva quanto jornalística (estudo de caso ilustrativo);

4. explorar as situações em que a intervenção a ser avaliada não apresenta um conjunto único e distinto de resultados (estudo de caso exploratório);

5. meta-avaliação: um estudo de avaliação (estudo de caso de meta-avaliação).

Além dessas cinco possíveis aplicações, os tipos de estudos de caso podem ser quatro, como mostrado na tabela a seguir.

Tabela n.1: Tipos de estudo de caso

\begin{tabular}{|l|c|c|}
\cline { 2 - 3 } \multicolumn{1}{c|}{} & Caso único & Casos múltiplos \\
\hline Holístico: unidade única de análise & Tipo 1 & Tipo 2 \\
\hline Incorporado: unidades múltiplas de análise & Tipo 3 & Tipo 4 \\
\hline
\end{tabular}

Fonte: YIN, 2005, p.61 
O projeto de pesquisa incluiu a definição de questões específicas de pesquisa, a escolha de testemunhas privilegiadas e o uso de múltiplas fontes de dados para se referir a uma maior variedade de temas, reconhecendo no desenvolvimento da convergência das linhas de pesquisa uma das vantagens mais importantes da metodologia do estudo de caso. $\mathrm{O}$ presente projeto baseia-se numa pesquisa prevalentemente qualitativa, mas prevendo a coleta de dados quantitativos e qualitativos através do uso de três princípios metodológicos como a seguir (YIN, 1985, pp. 102, 103):

\subsection{PRINCÍPIOS METODOLÓGICOS DE COLETA DE DADOS}

a) Utilização de várias fontes de evidência, e não apenas uma;

b) A criação de um banco de dados para o estudo de caso; e

c) A manutenção de um encadeamento de evidências.

\subsection{FONTES DE EVIDÊNCIAS}

5.2.1 Documentos (estatutos, projetos, convenções, monografias de graduação, relatórios, regulamentos, leis, relatórios de arquivo, artigos, tabelas, gráficos, diários, sites, e-mail, fotos e vídeos): Para os estudos de caso, o uso mais importante da documentação é validar e apoiar evidências de outras fontes.

5.2.2 Observação participante: Nasce na pesquisa antropológica e refere-se em particular à abordagem etnográfica. Para o envolvimento do pesquisador no contexto estudado e sua interação com os atores sociais, ele é colocado em um paradigma interpretativo. Nessa perspectiva, o envolvimento e a identificação não devem ser evitados e a subjetividade da interação e interpretação é uma das principais características. Além disso, como sugerem Amaturo (2012) e Corbetta (2014), o pesquisador insere-se: diretamente por um período de tempo relativamente longo em um determinado grupo social; em seu ambiente natural, estabelecendo uma relação de interação pessoal com seus membros; Para descrever as ações e compreender as motivações através de um processo de identificação.

5.2.3 Diário de campo: O segundo dos três princípios de Yin (1985), relacionados à coleta de dados, a saber: "a criação de um banco de dados para o Estudo de Caso", refere-se à organização e documentação dos dados coletados que podem fornecer duas coleções 
separadas: a primeira de dados ou base de evidências e a segunda um diário / relatório do pesquisador na forma.

5.2.4 Questionários: Considera-se que um questionário é constituído por uma lista estruturada de perguntas, algumas das quais estão fechadas e outras abertas. As perguntas fechadas fornecem alternativas de resposta pré-definidas (pré-codificação) que usam escalas de resposta (TRINCHERO, p.33). Quanto às questões abertas, uma vez que não há pré codificação de possíveis respostas (relacionadas a atitudes, opiniões, orientações, percepções, emoções, motivações, juízos de valor e até avaliações), a análise das respostas fornece uma pós-codificação com as respostas. atribuição de categorias específicas (CORBETTA, 2014, p.174).

5.2.5 Entrevistas: Uma das mais importantes fontes de informações para um estudo de Caso (YIN, 1985, pag.112). As entrevistas, de tipo semiestruturadas, incluem um roteiro com o número de questões que pode variar. Podem ser conduzidas de forma espontânea, permitindo que o pesquisador tanto indague 'respondentes-chave' sobre os fatos de uma maneira quanto peça a opinião deles sobre determinados eventos. Em algumas situações, pode-se até mesmo pedir que o respondente apresente suas próprias interpretações de certos acontecimentos e pode-se usar essas proposições como base para uma nova pesquisa.

O estudo de caso apresentado neste projeto é pensado e planejado como um estudo de caso único, incorporado e descritivo para poder, de fato, descrever o objeto de investigação no momento em que ocorre. Zainal (2007, p.3) sugere que estudos de caso descritivos também podem ser desenvolvidos na forma narrativa.

O estudo descritivo examina o campo e a profundidade do objeto descrito. Um estudo de caso descritivo, portanto, apresenta uma descrição completa de um fenômeno dentro de seu contexto. Quanto mais a abordagem teórica do fenômeno for atendida, melhor será o estudo do caso descritivo. Mas onde a descrição começa e onde termina, o que inclui e o que a descrição de um possível objeto de estudo de caso deve ser excluída? Os critérios utilizados para responder a essas perguntas estão contidas no mesmo "desenho de pesquisa" do estudo de caso. "(CEDE, 2001, p.10-11).

Dada a complexidade do objeto da pesquisa, decidiu-se abordar o estudo de caso em questão por meio de duas subunidades de análise como a seguir. 


\subsection{SUBUNIDADES DE ANÁLISE}

1. A implementação do Ciclo INTEREURISLAND no PPGESA, DCH III, UNEB, no período entre junho de 2018 e Abril de 2019.

2. A relevância da educação contextualizada no desenvolvimento da educação Glocal e vice-versa, através da implementação do Ciclo na subunidade n.1.

\subsection{HIPÓTESES DE PESQUISA}

1. É possível implementar o Ciclo INTEREURISLAND no PPGESA, DCH III, UNEB, no período entre junho de 2018 e Maio de 2020;

2. A Educação contextualizada é de grande relevância na implementação do Ciclo INTEREURISLAND no PPGESA e no desenvolvimento da educação GloCal, e vice-versa.

3. A educação contextualizada para a convivência com o semiárido traz em si princípios que podem ser extrapolados e vivenciados em quaisquer outros contextos educacionais.

No projeto de pesquisa elaborado, segundo o cronograma, cada subunidade de análise apresentará um protocolo de pesquisa que descreve as questões específicas de pesquisa, as fontes, as ferramentas, o cronograma de coleta de dados e os métodos de análise dos mesmos.

A figura $n .4$, a seguir, nos propõe uma visualização gráfica da implementação do Ciclo INTEREURISLAND no PPGESA, que ressalta as duas hipóteses de pesquisa colocando a Educação contextualizada como 'Foco central' de intersecção das três áreas de interesse. 
Figura n.4: O Ciclo INTEREURISLAND e a Educação Contextualizada.

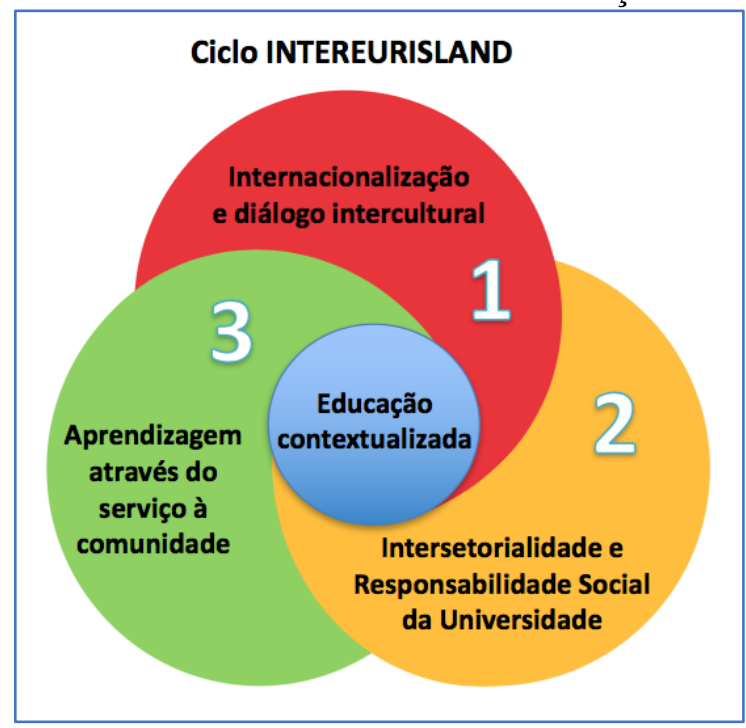

Na tabela $n .2$ a seguir são apresentadas as atividades específicas de cada área do Ciclo INTEREURISLAND, para ampliar o entendimento da metodologia a ser implementada, tendo em vista seu desenvolvimento e contribuição para a pós-graduação do DCH III.

\section{Tabela n.2: Atividades do Ciclo INTEREURISLAND} Internacionalização e diálogo inter-cultural

\section{Criação, desenvolvimento e institucionalização de parcerias internacionais}

a) Estipulação de acordos bilaterais de colaboração acadêmica (Memorando de Entendimento - MoU), entre as universidades envolvidas;

b) Estipulação da Adenda ao Memorando (MoU), entre os departamentos / faculdades das áreas de interesse;

c) Estipulação de acordos de co-tutoria para tese de doutoramento entre universidades.

\section{Mobilidade Estudantil Internacional (IN \& OUT)}

a) IN: Recepção e orientação para atividades de estudo + estágio (extensão) e pesquisa;

b) IN: certificação / reconhecimento de atividades e créditos;

c) OUT: orientação para a proposta INTEREURISLAND

d) OUT: seleção e definição do Contrato de Aprendizagem (atividade de estudo + estágio e / ou pesquisa a ser desenvolvida no exterior)

e) OUT: reconhecimento de crédito (pós-mobilidade).

3 Mobilidade internacional de pesquisadores, professores e pessoal técnico-administrativo (IN \& OUT)

Pesquisadores - Docentes - Pessoal técnico
a) Atividade de ensino
b) Pesquisa
c) Extensão (projeto / programas integrados)
d) Seminários, conferências - Publicações

a) Atividades interculturais (idiomas inclusos)

4 Formação específica

b) Educação entre pares

5 Avaliação em andamento e final

6 Planejamento do novo ciclo INTEREURISLAND 
Intersetorialidade e responsabilidade social da universidade

\section{Criação, desenvolvimento e institucionalização de parcerias locais}

a) Estipulação de acordos entre as universidades, departamentos / faculdades envolvidas e a comunidade: órgãos públicos e privados, grupos formais, não formais e informais.

b) Criação do grupo de trabalho intersetorial: professores / tutores acadêmicos, pesquisadores, tutores / representantes da comunidade, estudantes locais e estrangeiros envolvidos;

c) Planejamento de projetos de engajamento social / público: entre departamentos / faculdades ou cursos de graduação e órgãos comunitários / grupos envolvidos;

d) Desenvolvimento de projetos de engajamento social / público: entre departamentos / faculdades ou cursos de graduação e órgãos comunitários / grupos envolvidos;

2 Desenvolvimento de oficinas educativas interativas:

a) No campo acadêmico (dentro dos cursos universitários de interesse com o povo da comunidade)

b) Dentro da comunidade (entre as atividades realizadas nos corpos / grupos da comunidade)

3 Avaliação em andamento e final

4 Planeamento do novo ciclo INTEREURISLAND

\section{Aprendizagem através do serviço solidário}

\section{1: Envolvimento e reciprocidade}

2: Escolha / definição de objetivos acadêmicos: para estudantes locais e estrangeiros, cursando cursos de pós-graduação de interesse e envolvidos em projetos de engajamento social / público com a comunidade;

3: Identificação das reais necessidades / desafios da comunidade: dos órgãos / grupos de referência envolvidos em projetos de engajamento social / público;

4: Planejamento das atividades do projeto: em colaboração contínua com a comunidade;

5: Implementação das atividades do projeto: em colaboração contínua com a comunidade;

6: reflexão sistemática sobre a experiência vivida;

7) avaliação: em andamento e final

8 Demonstração de novas aprendizagens e resultados obtidos;

9 Planejamento do novo ciclo INTEREURISLAND.

Fonte: Andrian N. (2017, pag.5)

\section{CONCLUSÕES}

O artigo apresentou a pesquisa de Pós-doutorado cujo título é "Do Local ao Global. A educação contextualizada e o diálogo intercultural na proposta de internacionalização da extensão INTEREURISLAND no PPGESA”. Uma pesquisa vinculada ao Programa de Pós-Graduação em Educação, Cultura e Territórios Semiáridos - PPGESA que, como fase sucessiva da pesquisa INTEREURISLAND ${ }^{50}$ (ANDRIAN, 2017), desenvolvida através de um doutorado em Co Tutela entre a UNIPD e a UNEB, abre os horizontes e o cenário de investigão, ação e formação à Educação Contextualizada como Focus, centro através do qual articulam-se as áreas de internacionalização e dialogo intercultural, responsabilidade social da universidade e aprendizagem solidária.

Um vertente de interesse tanto pelo PPGESA como por ambas as universidades envolvidas nos processos de pesquisa e intercâmbio e, também , pela Coordenação de

\footnotetext{
50 Título completo: "INTEREURISLAND. Da uma pesquisa no campo a uma possível referência para a internacionalização de experiências de responsabilidade social da Universidade"
} 
Aperfeiçoamento de Pessoal de Nível Superior - CAPES, que aprovou o projeto apresentado na chamada pública de Propostas de eventos científicos, no âmbito do Programa de Apoio a Eventos no País - PAEP (edital nº 29/2018).

O PAEP tem como objetivos específicos: Apoiar a divulgação da produção científica e tecnológica incentivando a inovação e a geração de conhecimentos, de parcerias e de produtos; Promover a melhoria da qualidade da produção científica e tecnológica nacional; Apoiar eventos que fortalecem a cooperação destinados à pós-graduação e parceiros internacionais e Incentivar a participação de professores e alunos de pós-graduação. (CAPES, 2018)

O evento científico aprovado, cujo título é "COLÓQUIO BRASIL ITÁLIA - A educação contextualizada e o diálogo intercultural na implementação de novas estratégias de internacionalização da extensão no PPGESA DCH III/UNEB”, realizou-se no Departamento de Ciências Humanas / DCH, Campus III, UNEB entre o dia 31 de Julho e o dia 23 de Agosto de 2019 e é o Tema do presente número específico da Revista de Comunicação e Cultura no Semiárido - ComSertões ${ }^{51}$.

\section{Referências bibliográficas}

AMATURO, Enrica. Metodologia della ricerca sociale. Bologna: UTET Università, 2012.

ANDRIAN, Nicola. INTERERISLAND: Una ricerca e un modello in merito a processi di Internazionalizzazione di esperienze di Responsabilità Sociale dell'Università. PLURAIS, Salvador-BA, v. 2, n. 2, p. 46-67, abr./ago. 2017.

CAPES. A internacionalização na Universidade Brasileira: resultados do questionário aplicado pela CAPES. Brasilia: Edição e composição: Diretoria de Relações Internacionais, 2017.

Chamada pública de propostas de eventos científicos no âmbito do Programa de Apoio a Eventos no País - PAEP. Edital nº 29/2018. Disponível ao link: https://www.capes.gov.br/images/stories/download/editais/14082018_Edital_29_PAEP. pdf

CEDE - Istituto Nazionale per la valutazione del sistema dell'istruzione. Guida metodologica agli Studi di Caso. Frascati Roma: Progetto QUASI, 2001.

CORBETTA, Piergiorgio. Metodologia e tecniche della ricerca sociale, $2^{\mathrm{a}} \mathrm{Ed}$., Bologna: Il Mulino, 2014.

${ }^{51}$ https://www.revistas.uneb.br/index.php/comsertoes 
FREIRE, Paulo. Pedagogia da tolerância. Ana Maria Araújo Freire (Org.). São Paulo: UNESP, 2005.

Conscientização e Alfabetização: uma nova visão do processo. Recife:

Revista de Cultura da Universidade do Recife. No 4; Abril-Junho, 1963.

FREIRE, Paulo \& FAUNDEZ, Antônio. Por uma Pedagogia da Pergunta. $3^{\text {a }}$ Ed. Rio de Janeiro: Paz e Terra, 1985.

FÓRUM DE PRÓ - REITORES DE EXTENSÃO DAS UNIVERSIDADES PÚBLICAS BRASILEIRAS. Política Nacional De Extensão Universitária: Recife, Editora Universitária, UFPE, 2013.

GALERI, Patrizia (A cura di). Ambientando. Riflessione pedagogica ed esperienze didattiche per l'ambiente. Milano: EDUCatt., 2009.

GEORGESCU, Mara. T-KIT 4 - Intercultural Learning 2ªdição. Strasbourg: COUNCIL OF EUROPE, 2018.

KÜSTER, Angela; MATTOS, Beatriz: Educação no contexto do semiárido brasileiro. Fortaleza: Fundação Adenauer Konrad, 2004.

MARTINS, Josemar da Silva. Anotações em torno do conceito de educação para a convivência com o semi-árido. In: Secretaria Executiva da RESAB. Educação para a convivencia com o Semi-arido: Reflexoes teorico-praticas. $2^{\text {a }}$ Ed., Juazeiro-BA: Selo editorial RESAB, 2006.

PEREIRA, Vanderléa Andrade. Multiplos olhares sobre a educação no Semiárido Brasileiro. Juazeiro-BA: Gráfica e Editora Printpex, Caderno Multidisciplinar educação e Contexto do Semiárido Brasileiro, Ano 10 n08, Dezembro, 2016.

PEROZA, Juliano. Reflexões sobre cultura e diversidade cultural em Paulo Freire: Um humanismo crítico para a transculturalidade em educação. Agência: CAPES, 2012.

REIS, Edmerson dos Santos. Educação para a convivência com o semiárido: desafios e possibilidades. in SOUSA e SILVA, Conceição de Maria de; LIMA, Elmo de Souza; CANTALICE, Maria Luiza de; ALENCAR, Maria Teresa de Alencar; SILVA, Walderene Alves Lopes da. Semiárido Piauiense .. Educação e contexto. Campina Grande-PB: INSA, 2010.

ROLAND, R. Globalization: Social Theory and Global Culture. London: SAGE Publications, 1992.

TRINCHE.RO, Roberto. I metodi della ricerca educative. Bari: Edizioni Laterza, 2004

YIN, Robert K.. Estudo de caso. Planejamento e Métodos. 2ªdição, São Paulo: BOOKMAN, 1994. 
SILVA, Ana Célia Da. A representação social do negro no livro didático: O que mudou? Por que mudou? Salvador: EDUFBA, 2011.

SMITT, Craig N.. The Case Study: a useful research method for information management. In: Journal of Information Technologies n.5, pp. 123 - 133, Palgrave Macmillan UK, 1990.

STAKE, R. E.. The art of case study research. Thousand Oaks, CA: Sage Publications, 1995.

UNESCO. Guidelines on Intercultural Education. Parigi, Section of Education for Peace and Human Rights, Division for the Promotion of Quality Education, Education Sector, 2006.

UNESCO. Educazione alla cittadinanza globale - Temi e obiettivi di apprendimento. Roma: Centro per la Cooperazione Internazionale, 2018.

ZAINAL, Z.. Case study as a research method, Journal of JurnalKemanusiaan, Vol.9, No. January, PP.1-6, 2007. 